Research Article

\title{
The Novel Successive Variational Mode Decomposition and Weighted Regularized Extreme Learning Machine for Fault Diagnosis of Automobile Gearbox
}

\author{
Yijiao Wang $(\mathbb{D}$ and Guoguang Zhou \\ College of Transportation Engineering, Chang'an University, Xi'an 710064, China \\ Correspondence should be addressed to Yijiao Wang; yijiao.wang@chd.edu.cn
}

Received 25 January 2021; Revised 5 February 2021; Accepted 16 February 2021; Published 24 February 2021

Academic Editor: Yi Qin

Copyright (c) 2021 Yijiao Wang and Guoguang Zhou. This is an open access article distributed under the Creative Commons Attribution License, which permits unrestricted use, distribution, and reproduction in any medium, provided the original work is properly cited.

\begin{abstract}
In order to improve the diagnosis accuracies of the current diagnosis methods, a novel fault diagnosis method of automobile gearbox based on novel successive variational mode decomposition and weighted regularized extreme learning machine is presented for fault diagnosis of gearbox in this paper. The novel successive variational mode decomposition (SVMD) is presented to improve the traditional variational mode decomposition, which finds modes one after the other, and this succession helps increase convergence rate and also not extract the unwanted modes; weighted regularized extreme learning machine (WRELM) is presented to improve the traditional extreme learning machine, which uses the weight of each sample with the nonparametric kernel density estimation and can find the optimal weight for each sample. The test results indicate that the diagnosis accuracy of SVMD-WRELM for gearbox is better than that of VMD-WRELM, VMD-ELM.
\end{abstract}

\section{Introduction}

Gearbox is the key component of automobile, and condition monitoring and fault diagnosis for automobile gearbox are very significant [1-4]. Recently, artificial neural networks (ANNs), support vector machine (SVM), etc. have been successfully applied to diagnose the faults of gearbox. For instance, Qiu et al. presented a deep convolutional neural network model for intelligent fault diagnosis of a gearbox under different operational conditions [5]. Jedliński and Jonak presented an early fault detection method in gearboxes based on support vector machines and multilayer perceptron with a continuous wavelet transform [6]. Extreme learning machine (ELM) is a simple and efficient single hidden layer feedforward network [7-9]. Compared with ANNs and SVM, ELM has better generalization ability [10], so ELM has a good application prospect in the field of fault diagnosis for gearbox. In order to improve the performance of ELM, weighted regularized extreme learning machine (WRELM) is the new extreme learning machine using the weight of each sample with the nonparametric kernel density estimation and can find the optimal weight for each sample. Thus, WRELM is used to classify the states of gearbox.

In addition, the feature extraction of the vibrational signal of gearbox has a great influence on the accuracy of fault diagnosis of gearbox. Recently, empirical mode decomposition (EMD), variational mode decomposition (VMD), etc. have been successfully applied to extract the features of gearbox. Hongmei Liu et al. applied empirical mode decomposition in the feature extraction of the vibrational signal of gearbox [11].Vikas Sharma and Anand Parey applied variational mode decomposition in the feature extraction of the vibrational signal of gearbox under varying speed [12]. There are problems such as mode mixing in EMD; VMD is a new multicomponent signal decomposition method based on Wiener filtering and Hilbert transform, which aims to make decomposition of a target signal into a set of modes with sparsity properties being achievable [13-15]. In order to improve the performance of VMD, 
successive variational mode decomposition (SVMD) finds modes one after the other, and this succession helps increase convergence rate and also not extract the unwanted modes.

Thus, in order to improve the diagnosis accuracies of the current diagnosis methods, a novel fault diagnosis method based on successive variational mode decomposition and weighted regularized extreme learning machine (SVMDWRELM) is presented for fault diagnosis of automobile gearbox in this paper. The four states of automobile gearbox including normal state, wear and tear, pitting fault, and snaggletooth fault are used in this experiment. The testing results indicate that the diagnosis accuracies of SVMDWRELM for gearbox are better than those of VMDWRELM, VMD-ELM.

\section{Successive Variational Mode Decomposition}

Variational mode decomposition can decompose the signal $f(t)$ into a set of VMDSs $u_{l}(t)$ around the center frequencies $\omega_{l}$ according to the following equation [16]:

$$
\min _{\left\{u_{l}\right\},\left\{\omega_{l}\right\}}\left(\sum_{l=1}^{L}\left\|\partial_{t}\left[\left(\rho(t)+\frac{j}{\pi t}\right) * u_{l}(t)\right] e^{-j \omega_{l} t}\right\|_{2}^{2}\right),
$$

subject to the conditions,

$$
\sum_{l=1}^{L} u_{l}(t)=f(t)
$$

where $\partial_{t}$ denotes the gradient operator, $*$ denotes the convolution operator, and $\rho(t)$ denotes the Dirac distribution.

The minimization problem of eq (1) is transformed into the following optimization problem:

$$
J\left(\left\{u_{l}\right\},\left\{\omega_{l}\right\}, \lambda\right)=\alpha \sum_{l=1}^{L}\left\|\partial_{t}\left[\left(\rho(t)+\frac{j}{\pi t}\right) * u_{l}(t)\right] e^{-j \omega_{l} t}\right\|_{2}^{2}+\left\|f(t)-\sum_{l=1}^{L} u_{l}(t)\right\|_{2}^{2}+\left\langle\lambda(t), f(t)-\sum_{l=1}^{L} u_{l}(t)\right\rangle,
$$

where $\alpha$ denotes the constraint factor and $\lambda(t)$ denotes the Lagrangian multiplier.

Successive variational mode decomposition is a method which finds modes one after the other, and this succession helps increase convergence rate and also not extract the unwanted modes. In successive variational mode decomposition, the combination of quadratic penalty term and Lagrangian multiplier can be used to establish the augmented Lagrangian function as

$$
\begin{aligned}
G\left(\left\{u_{l}\right\},\left\{\omega_{l}\right\}, \lambda\right)= & \alpha \sum_{l=1}^{L}\left\|\partial_{t}\left[\left(\rho(t)+\frac{j}{\pi t}\right) * u_{l}(t)\right] e^{-j \omega_{l} t}\right\|_{2}^{2}+\left\|f(t)-\sum_{l=1}^{L} u_{l}(t)\right\|_{2}^{2}+\left\langle\lambda(t), f(t)-\sum_{l=1}^{L} u_{l}(t)\right\rangle \\
& +\left\|f(t)-\left(u_{L}(t)+f_{u}(t)+\sum_{l=1}^{L-1} u_{l}(t)\right)\right\|_{2}^{2}+\left\langle\lambda(t), f(t)-\left(u_{L}(t)+f_{u}(t)+\sum_{l=1}^{L-1} u_{l}(t)\right)\right\rangle .
\end{aligned}
$$

After using Parseval's equality, eq (4) is transformed into

$$
\begin{aligned}
& G\left(\left\{u_{l}\right\},\left\{\omega_{l}\right\}, \lambda\right)=\alpha\left\|j\left(\omega-\omega_{L}\right)\left[(1+\operatorname{sgn}(\omega)) \tilde{u}_{L}(\omega)\right]\right\|_{2}^{2}+\left\|\tilde{\gamma}_{L}(\omega)\left(\tilde{f}_{u}(\omega)+\sum_{l=1}^{L-1} \widetilde{u}_{l}(\omega)\right)\right\|_{2}^{2}+\sum_{l=1}^{L-1}\left\|\tilde{\gamma}_{l}(\omega) \cdot u_{l}(\omega)\right\|_{2}^{2} \\
& +\left\|\tilde{f}(\omega)-\left(\tilde{u}_{L}(\omega)+\tilde{f}_{u}(\omega)+\sum_{l=1}^{L-1} \tilde{u}_{l}(\omega)\right)\right\|_{2}^{2}+\left\langle\tilde{\lambda}(\omega), \tilde{f}(\omega)-\left(\tilde{u}_{L}(\omega)+\tilde{f}_{u}(\omega)+\sum_{l=1}^{L-1} \widetilde{u}_{l}(\omega)\right)\right\rangle . \\
& \text { By letting the first variation vanish for the positive } \quad \widetilde{u}_{L}^{n+1}(\omega)=\frac{\tilde{f}(\omega)-\left(\widetilde{f}_{u}^{n}(\omega)+\sum_{l=1}^{L-1} \widetilde{u}_{l}(\omega)\right)+(\tilde{\lambda}(\omega) / 2)}{1+2 \alpha\left(\omega-\omega_{L}^{n}\right)^{2}+\sum_{l=1}^{L-1}\left(1 / \alpha^{2}\left(\omega-\omega_{l}\right)^{4}\right)^{2}} .
\end{aligned}
$$
frequencies by minimizing eq (5), 
By minimizing eq.(6), $\tilde{f}_{u}^{n+1}(\omega)$ will be described as

$$
\tilde{f}_{u}^{n+1}(\omega)=\frac{\alpha^{2}\left(\omega-\omega_{L}^{n+1}\right)^{4}\left(\tilde{f}(\omega)-\left(\tilde{f}_{u}^{n}(\omega)+\sum_{l=1}^{L-1} \widetilde{\mathfrak{u}}_{l}(\omega)\right)+(\tilde{\lambda}(\omega) / 2)\right)-\sum_{l=1}^{L-1} \widetilde{\mathfrak{u}}_{l}(\omega)}{1+\alpha^{2}\left(\omega-\omega_{L}^{n+1}\right)^{4}} .
$$

The equation for updating the Lagrangian multiplier $\lambda$ is achieved by the dual ascent method:

$$
\tilde{\lambda}^{n+1}=\tilde{\lambda}^{n}+\tau\left[\tilde{f}(\omega)-\left(\widetilde{u}_{L}^{n+1}(\omega)+\tilde{f}_{u}^{n}(\omega)+\sum_{l=1}^{L-1} \widetilde{u}_{l}(\omega)\right)\right],
$$

where $\tau$ denotes the update parameter.

By replacing $\widetilde{f}_{u}^{n+1}(\omega)$ with eq $(8), \widetilde{\lambda}^{n+1}$ will be described as

$$
\tilde{\lambda}^{n+1}=\tilde{\lambda}^{n}+\tau\left[\tilde{f}(\omega)-\left(\tilde{u}_{L}^{n+1}(\omega)+\frac{\alpha^{2}\left(\omega-\omega_{L}^{n+1}\right)^{4}\left(\tilde{f}(\omega)-\widetilde{u}_{L}^{n+1}(\omega)-\sum_{l=1}^{L-1} \widetilde{u}_{l}(\omega)\right)-\sum_{l=1}^{L-1} \widetilde{u}_{l}(\omega)}{1+\alpha^{2}\left(\omega-\omega_{L}^{n+1}\right)^{4}}+\sum_{l=1}^{L-1} \widetilde{u}_{l}(\omega)\right)\right] .
$$

As shown in Figures 1 and 2, the six decomposition signals (SVMDSs) described as SVMDS1 SVMDS6 of the vibrational signal of gearbox are obtained based on SVMD. The features of the vibrational signal of gearbox are obtained by calculating the Shannon entropy of SVMDS1 SVMDS6. In Figure 1, abscissa values are the sampling data, and ordinate values are the amplitudes of the vibrational signal of gearbox; in Figure 2, abscissa values are the sampling data, and ordinate values are the amplitudes of SVMDS1 SVMDS6 of the vibrational signal of gearbox, respectively.

Figure 3 gives the comparison among the feature values of normal state of the vibrational signal of gearbox obtained by SVMD, the feature values of fault state of the vibrational signal of gearbox obtained by SVMD, the feature values of normal state of the vibrational signal of gearbox obtained by $\mathrm{VMD}$, and the feature values of fault state of the vibrational signal of gearbox obtained by VMD, and abscissa values are the six features and ordinate values are the values of the six features, which indicates that there are more obvious distinctions between the feature values of normal state and fault state of gearbox by SVMD than those by VMD.

\section{Weighted Regularized Extreme Learning Machine}

Assume $N$ hidden nodes in extreme learning machine and a sample pair $\left\{X_{i}, P_{i}\right\}$, and the output of extreme learning machine can be expressed as followings:

$$
f\left(X_{i}\right)=\sum_{n=1}^{N} \alpha_{n} H\left(\varphi_{n}, X_{i}, b_{n}\right), \quad i=1,2, \ldots, N,
$$

where $H(\cdot)$ denotes the activation function, $\varphi_{n}$ denotes the input weight vector, $\alpha_{n}$ denotes the $n$th output weight, and $b_{n}$ denotes the corresponding bias.

$$
\widetilde{P}=H \alpha=\left[\begin{array}{cccc}
H\left(\varphi_{1}, X_{1}, b_{1}\right) & H\left(\varphi_{2}, X_{1}, b_{2}\right) & \cdots & H\left(\varphi_{N}, X_{1}, b_{N}\right) \\
H\left(\varphi_{1}, X_{2}, b_{1}\right) & H\left(\varphi_{2}, X_{2}, b_{2}\right) & \cdots & H\left(\varphi_{N}, X_{2}, b_{N}\right) \\
\vdots & \vdots & \vdots & \vdots \\
H\left(\varphi_{1}, X_{N}, b_{1}\right) & H\left(\varphi_{2}, X_{N}, b_{2}\right) & \cdots & H\left(\varphi_{N}, X_{N}, b_{N}\right)
\end{array}\right] \cdot \alpha,
$$

where $\widetilde{P}$ denotes the output vector.

In order to get the estimation for the parameter $\alpha$, the objective function is used as following:

$$
\arg \min \|\widetilde{P}-P\|_{2}^{2}=\arg \min \|H \alpha-P\|_{2}^{2},
$$

where $P$ denotes the observed data.

The objective function of weighted regularized extreme learning machine is expressed as

$$
\arg \min C\|\sigma \varepsilon\|_{2}^{2}+\|\alpha\|_{2}^{2},
$$

subject to the conditions,

$$
P=H \alpha+\varepsilon,
$$

where $\sigma$ denotes a diagonal matrix, $\varepsilon$ denotes the vector of regression error, and $C$ denotes a regularization term.

Lagrange multipliers are introduced to solve the above minimization problem, and the corresponding solution for $\alpha$ is given as 


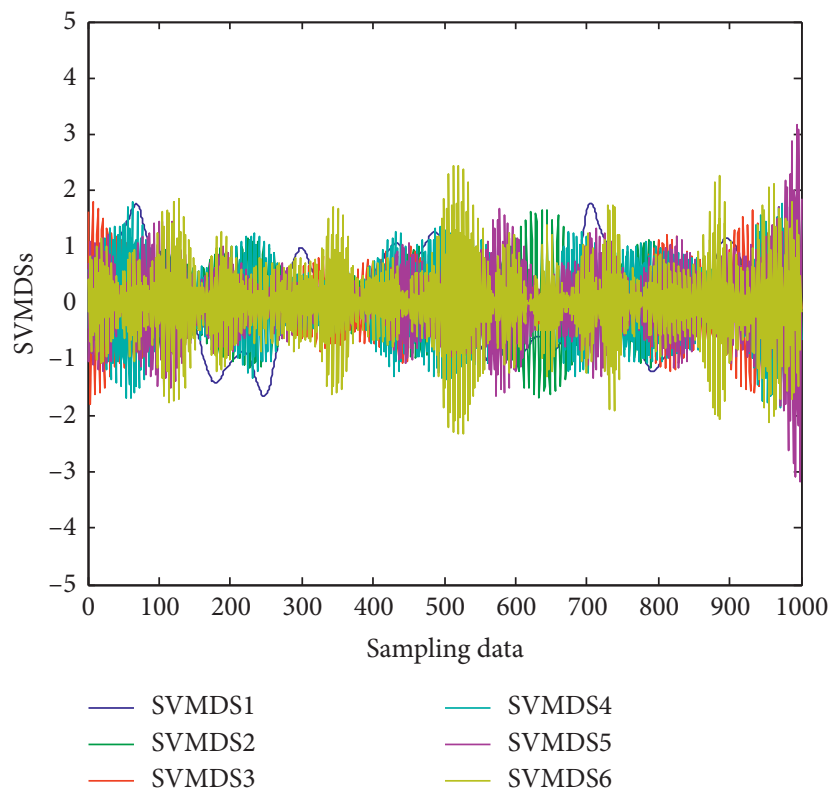

FIGURE 1: The decomposition of the vibrational signal of gearbox based on SVMD.

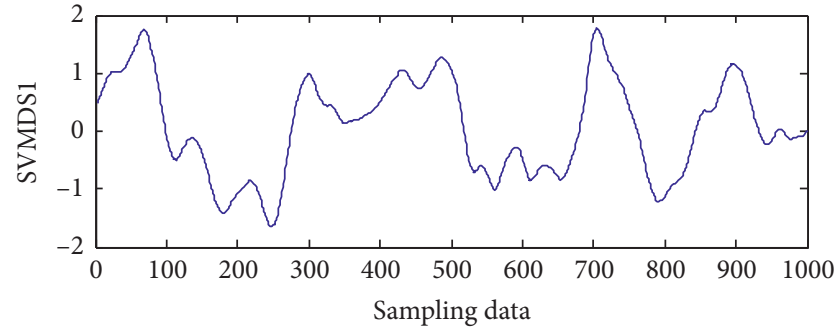

(a)

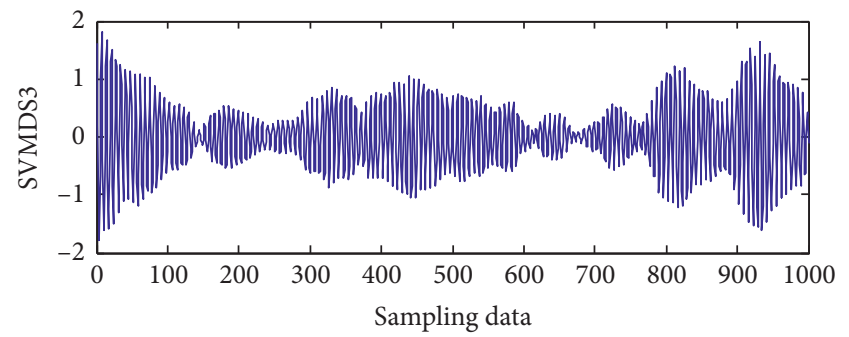

(c)

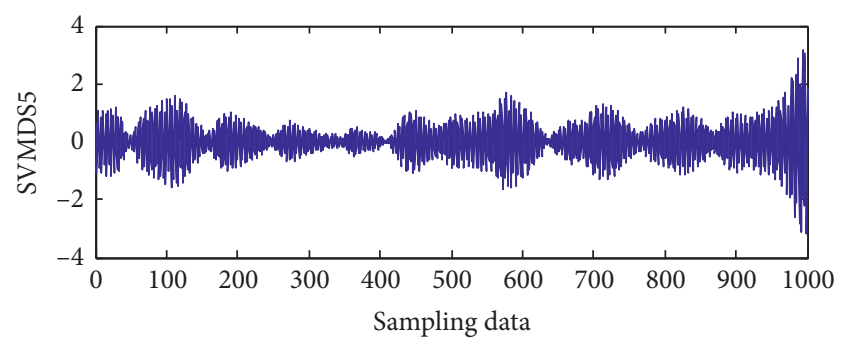

(e)

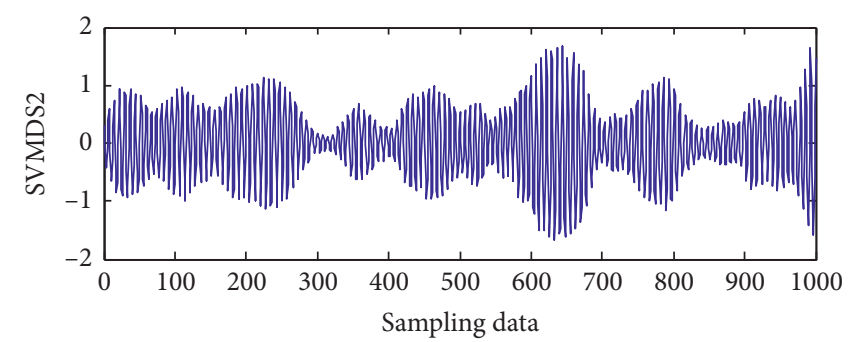

(b)

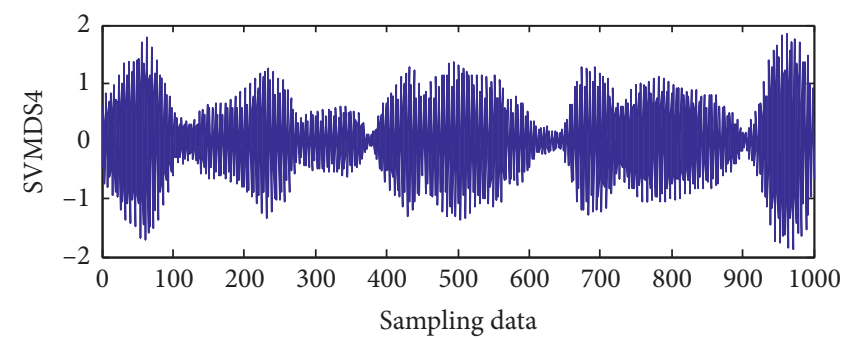

(d)

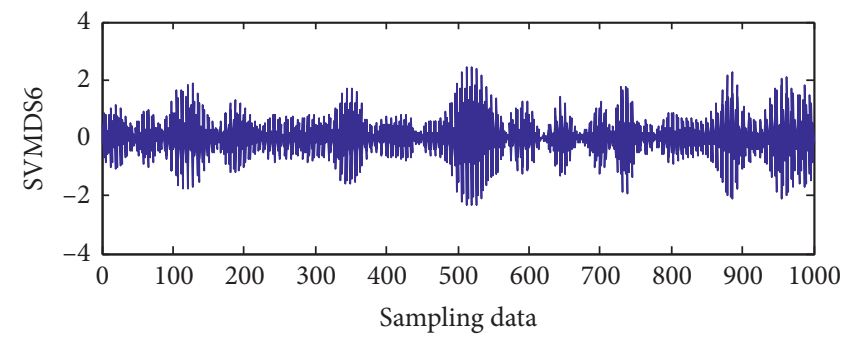

(f)

Figure 2: Six SVMDs. 

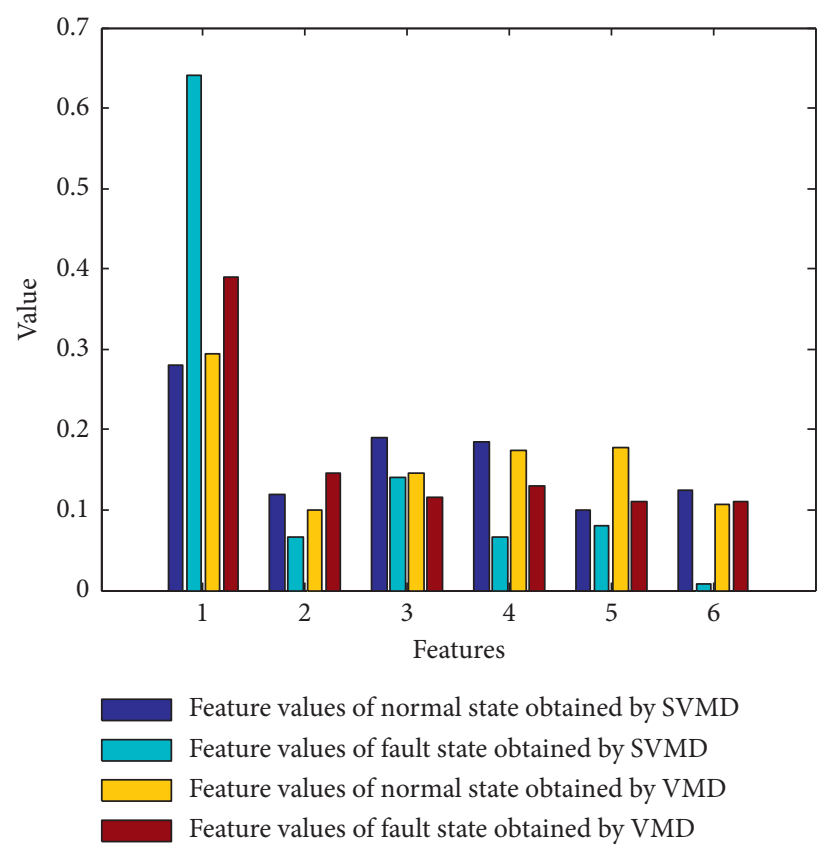

FIGURE 3: The comparison among the feature values of normal state of the vibrational signal of gearbox obtained by SVMD, the feature values of fault state of the vibrational signal of gearbox obtained by SVMD, the feature values of normal state of the vibrational signal of gearbox obtained by VMD, and the feature values of fault state of the vibrational signal of gearbox obtained by VMD.

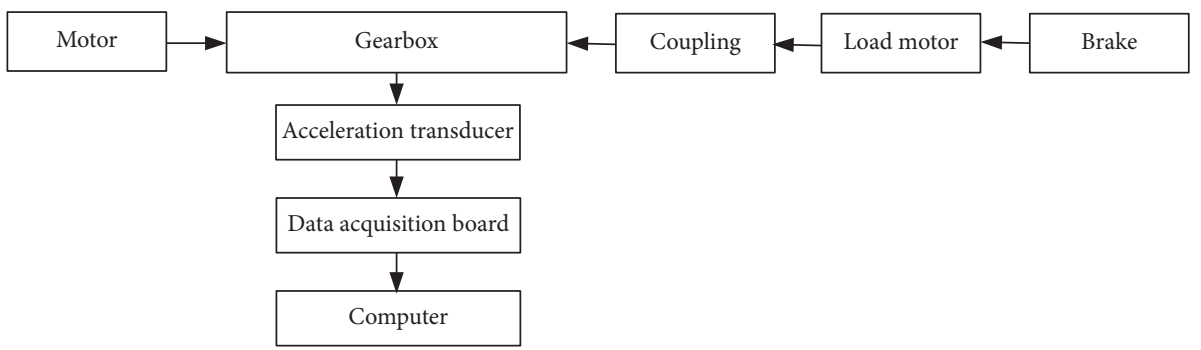

Figure 4: Framework of fault diagnosis for gearbox.

$$
\alpha=\left(H^{\prime} \sigma^{2} H+\frac{I}{C}\right)^{-1} H^{\prime} \sigma^{2} P
$$

\section{Experimental Analysis}

Figure 4 gives the framework of fault diagnosis for gearbox, which includes motor, coupling, gearbox, acceleration transducer, load motor, brake, data acquisition board, and computer. The vibrational signals of gearbox are measured with acceleration transducer, and the vibrational signals of gearbox are obtained by data acquisition board. Sampling frequency is $10 \mathrm{KHz}$, and the load is 1 N.m. The common faults of gearbox are wear and tear, pitting fault, and snaggletooth fault: wear and tear indicates the loss, injury of tooth surface due to suffering from pressure in the process of operation; pitting fault indicates local corrosion of tooth surface due to fatigue of tooth surface; and snaggletooth fault indicates crack of tooth.

The four states of gearbox including normal state, wear and tear, pitting fault, and snaggletooth fault are used in this experiment, among which normal state is denoted as state 1 of gearbox, wear and tear is denoted as state 2 of gearbox, pitting fault is denoted as state 3 of gearbox, and snaggletooth fault is denoted as state 4 of gearbox. 320 training samples include 80 samples denoting state 1 of gearbox, 80 samples denoting state 2 of gearbox, 80 samples denoting state 3 of gearbox, and 80 samples denoting state 4 of gearbox. 200 testing samples include 50 samples denoting state 1 of gearbox, 50 samples denoting state 2 of gearbox, 50 samples denoting state 3 of gearbox, and 50 samples denoting state 4 of gearbox. There is no intersection between the 320 training samples and the 200 testing samples.

The flow chart of fault diagnosis method based on SVMD-WRELM is described in Figure 5. In the experiment, the running speed of VMD for the vibrational signal of a sample of gearbox is about $0.75 \mathrm{~s}$, and the running speed of SVMD for the vibrational signal of a sample of gearbox is about $0.35 \mathrm{~s}$, which testifies the fast convergence of SVMD.

Several repeated experiments were performed, and the experimental results were identical. The comparison between actual results and diagnosis results for gearbox by 


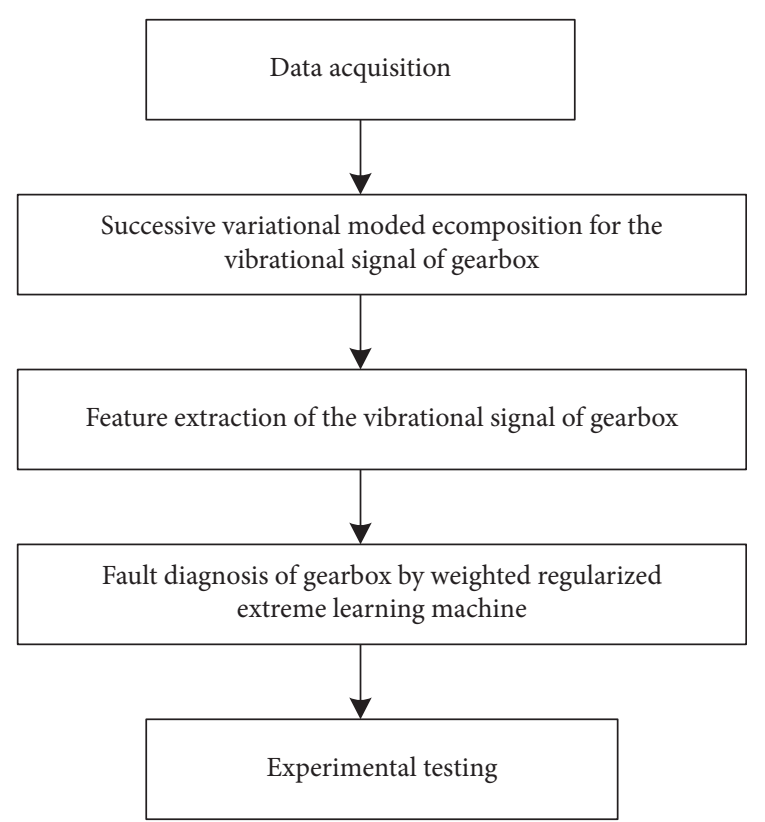

FIGURE 5: The flow chart of fault diagnosis method based on SVMD-WRELM.

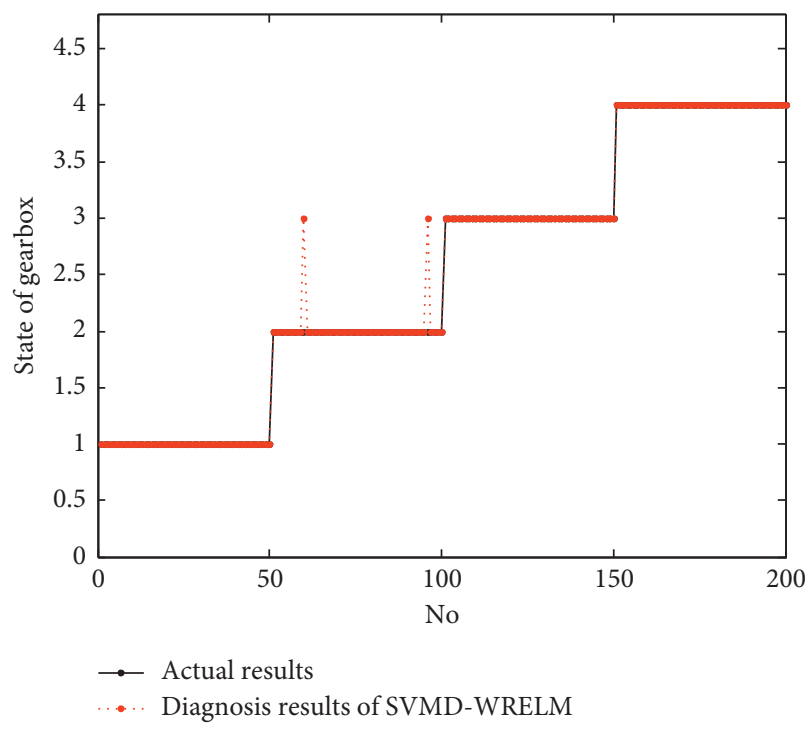

FIGURE 6: The comparison between actual results and diagnosis results for gearbox by SVMD-WRELM.

SVMD-WRELM are given in Figure 6, and the diagnosis accuracy of SVMD-WRELM for gearbox is $99 \%$. The comparison between actual results and diagnosis results for gearbox by VMD-WRELM is given in Figure 7, and the diagnosis accuracy of VMD-WRELM for gearbox is $97 \%$. The comparison between actual results and diagnosis results for gearbox by VMD-ELM is given in Figure 8, and the diagnosis accuracy of VMD-ELM for gearbox is $93.5 \%$. In Figures 6-8, abscissa values are the sample number, and ordinate values are the four states including normal state,

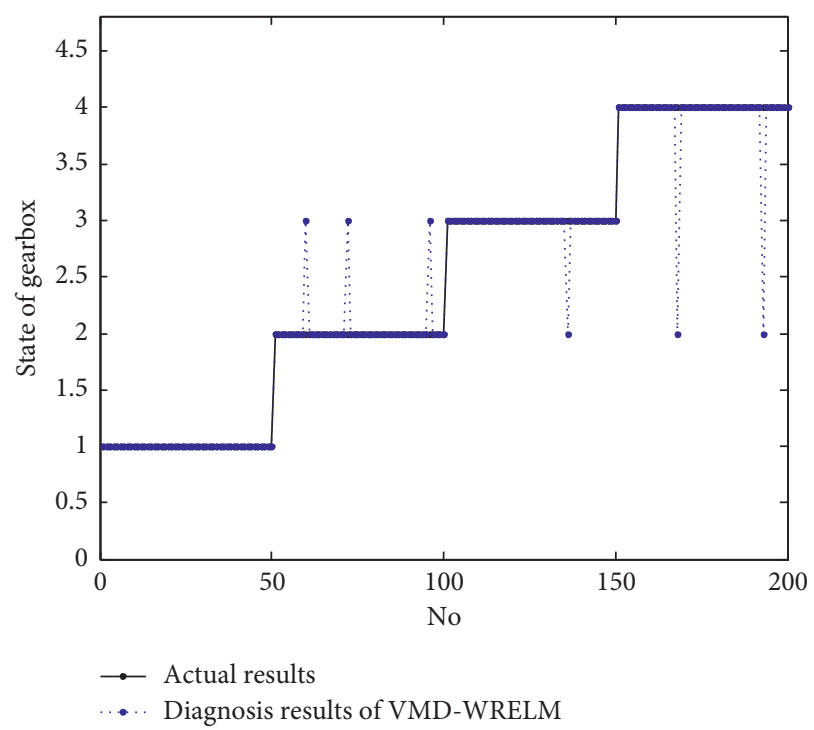

FIgURE 7: The comparison between actual results and diagnosis results for gearbox by VMD-WRELM.

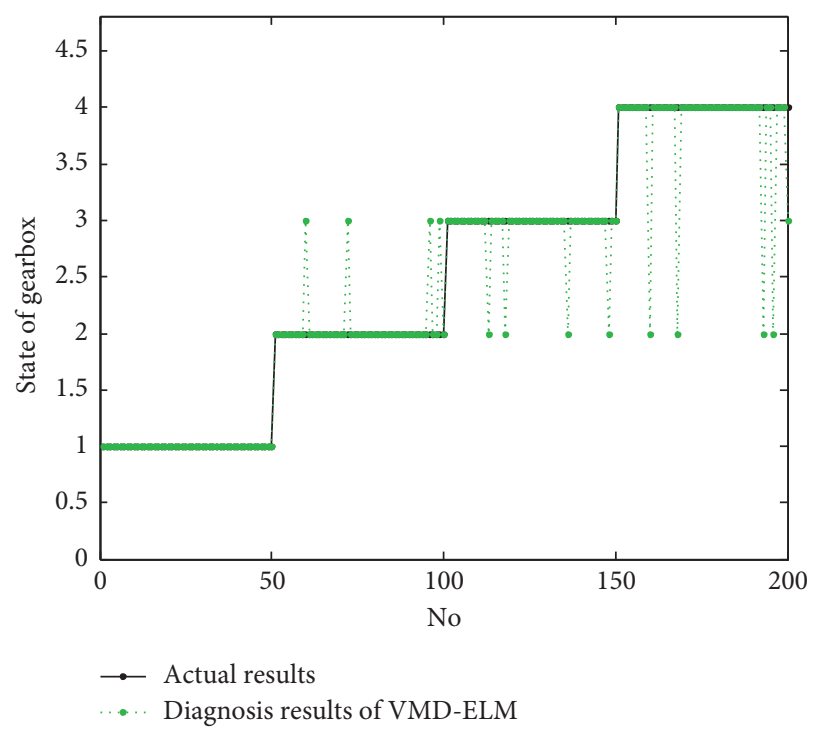

FIgURE 8: The comparison between actual results and diagnosis results for gearbox by VMD-ELM.

wear and tear, pitting fault, and snaggletooth fault, among which normal state is denoted as state 1 of gearbox, wear and tear is denoted as state 2 of gearbox, pitting fault is denoted as state 3 of gearbox, and snaggletooth fault is denoted as state 4 of gearbox.

As shown in Table 1, the diagnosis accuracies of SVMDWRELM for gearbox are better than those of VMDWRELM,VMD-ELM, and the diagnosis accuracies of VMDWRELM are better than those of VMD-ELM. It can be seen that SVMD is superior to VMD, and WRELM is superior to ELM. Therefore, fault diagnosis for gearbox by SVMDWRELM is feasible. 
TABLE 1: The comparison of the diagnosis accuracies among SVMD-WRELM, VMD-WRELM, and VMD-ELM.

\begin{tabular}{lccc}
\hline Diagnosis method & The total number of the training samples & The number of the corrected diagnosis & Diagnosis accuracy (\%) \\
\hline SVMD-WRELM & 200 & 198 & 99 \\
VMD-WRELM & 200 & 194 & 97 \\
VMD-ELM & 200 & 187 & 93.5 \\
\hline
\end{tabular}

\section{Conclusions}

A novel fault diagnosis method based on SVMD and WRELM is presented for fault diagnosis of automobile gearbox in this paper. SVMD finds modes one after the other, and this succession helps increase convergence rate and also not extract the unwanted modes, and WRELM uses the weight of each sample with the nonparametric kernel density estimation and find the optimal weight for each sample. The diagnosis accuracy of SVMD-WRELM for gearbox is $99 \%$, the diagnosis accuracy of VMD-WRELM for gearbox is $97 \%$, and the diagnosis accuracy of VMD-ELM for gearbox is $93.5 \%$. The testing results indicate that SVMD is superior to VMD, and WRELM is superior to ELM. It can be concluded that the diagnosis accuracies of SVMDWRELM for gearbox are better than those of VMDWRELM,VMD-ELM.

\section{Data Availability}

The data that support the findings of the research are available from the corresponding author.

\section{Conflicts of Interest}

The authors declare that there are no conflicts of interest regarding the publication of this article.

\section{Acknowledgments}

This study was supported by MOE (Ministry of Education in China) Project of Humanities and Social Sciences (grant no. 19YJA79097).

\section{References}

[1] I. Vamsi, G. R. Sabareesh, and P. K. Penumakala, "Comparison of condition monitoring techniques in assessing fault severity for a wind turbine gearbox under non-stationary loading," Mechanical Systems and Signal Processing, vol. 124, pp. 1-20, 2019.

[2] D. Dabrowski, "Condition monitoring of planetary gearbox by hardware implementation of artificial neural networks," Measurement, vol. 91, pp. 295-308, 2016.

[3] Y. Han, B. Tang, and L. Deng, "An enhanced convolutional neural network with enlarged receptive fields for fault diagnosis of planetary gearboxes," Computers in Industry, vol. 107, pp. 50-58, 2019.

[4] X. Chen and Z. Feng, "Time-frequency space vector modulus analysis of motor current for planetary gearbox fault diagnosis under variable speed conditions," Mechanical Systems and Signal Processing, vol. 121, pp. 636-654, 2019.

[5] G. Qiu, Y. Gu, and Q. Cai, "A deep convolutional neural networks model for intelligent fault diagnosis of a gearbox under different operational conditions," Measurement, vol. 145, pp. 94-107, 2019.

[6] Ł. Jedliński and J. . Jonak, "Early fault detection in gearboxes based on support vector machines and multilayer perceptron with a continuous wavelet transform," Applied Soft Computing, vol. 30, pp. 636-641, 2015.

[7] X. Wang, K. Yang, and J. H. Kalivas, "Comparison of extreme learning machine models for gasoline octane number forecasting by near-infrared spectra analysis," Optik, vol. 200, Article ID 163325, 2020.

[8] K. Akyol, "Comparing of deep neural networks and extreme learning machines based on growing and pruning approach," Expert Systems with Applications, vol. 140, Article ID 112875, 2020.

[9] Z. Chen, K. Gryllias, and W. Li, "Mechanical Fault diagnosis using convolutional neural networks and extreme learning machine," Mechanical Systems and Signal Processing, vol. 133, Article ID 106272, 2019.

[10] S. M. Naik, R. P. K. Jagannath, and V. Kuppili, "Fractional Tikhonov regularization to improve the performance of extreme learning machines," Physica A: Statistical Mechanics and Its Applications, vol. 551, Article ID 124034, 2020.

[11] H. Liu, J. Zhang, Y. Cheng, and C. Lu, "Fault diagnosis of gearbox using empirical mode decomposition and multifractal detrended cross-correlation analysis," Journal of Sound and Vibration, vol. 385, pp. 350-371, 2016.

[12] V. Sharma and P. Anand, "Extraction of weak fault transients using variational mode decomposition for fault diagnosis of gearbox under varying speed," Engineering Failure Analysis, vol. 107, Article ID 104204, 2020.

[13] Z. Xu, C. Li, and Y. Yang, "fault diagnosis of rolling bearing of wind turbines based on the variational mode decomposition and deep convolutional neural networks," Applied Soft Computing, vol. 95, Article ID 106515, 2020.

[14] Li Jin, X. Zhang, and J. Tang, "Noise suppression for magnetotelluric using variational mode decomposition and detrended fluctuation analysis," Journal of Applied Geophysics, vol. 180, Article ID 104127, 2020.

[15] C. Jayakumar and J. Sangeetha, "Kernellized support vector regressive machine based variational mode decomposition for time frequency analysis of mirnov coil," Microprocessors and Microsystems, vol. 75, Article ID 103036, 2020.

[16] G. Quaranta and F. Mollaioli, "Analysis of near-fault pulselike seismic signals through variational mode decomposition technique," Engineering Structures, vol. 193, pp. 121-135, 2019. 